\title{
Erratum
}

\section{Connecting Solutions of the Lorentz Force Equation do Exist}

\author{
E. Minguzzi ${ }^{1,2}$, M. Sánchez ${ }^{3}$ \\ 1 Departamento de Matemáticas, Plaza de la Merced 1-4, 37008 Salamanca, Spain. \\ E-mail: ettore.minguzzi@unifi.it \\ 2 INFN, Piazza dei Caprettari 70, 00186 Roma, Italy \\ 3 Departamento de Geometría y Topología Facultad de Ciencias, Avda. Fuentenueva s/n. 18071 Granada, \\ Spain. E-mail: sanchezm@ugr.es
}

Received: 5 May 2006 / Accepted: 5 May 2006

Published online: 5 August 2006 - (C) Springer-Verlag 2006

Commun. Math. Phys. 264, 349-370 (2006)

Due to a processing error the last paragraph of Sect. 3.1 on p. 358 was printed with an error. In addition, in Sect. 5.2 on pp. 365-366 the presentation of the Example was processed incorrectly. The second paragraph of Sect. 5.2 (two lines) must be removed and the last sentence in the same section must be replaced with 'Nevertheless, even though $\sigma_{0}$ maximizes in $\bar{C}_{1}$, it does not maximize in $\bar{C}_{2}$ (nor in the causal homotopy class $C$ ), in agreement with our results.' The corrected paragraphs read as follows.

\section{p. 358:}

In particular, if $x_{0} \ll x_{1}$ the two points can be connected by means of a timelike geodesic (in fact, by one for each time like homotopy class in $\mathcal{C}_{x_{0}, x_{1}}$, as will be apparent below). If $x_{1} \in E^{+}\left(x_{0}\right)=J^{+}\left(x_{0}\right) \backslash I^{+}\left(x_{0}\right)$ then $x_{0}$ and $x_{1}$ can still be joined by a lightlike geodesic, but this case does not make sense for the LFE. One can also wonder for the connectedness of $x_{0}, x_{1}$ by means of a geodesic even if they are not causally related, as in variational frameworks described below. Although this question has a geometrical interest (see for instance the survey [37]), it does not have a direct physical interpretation, nor equivalence for LFE.

pp. 365-366:

5.2. A remarkable example. Lemma 5.1 does not forbid the existence of a lightlike geodesic $\sigma$ which maximizes the functional on the closure of a timelike class $\overline{\mathcal{C}}_{x_{0}, x_{1}}$. However, in that case the maximizer on $\mathcal{C}_{x_{0}, x_{1}} \supset \overline{\mathcal{C}}_{x_{0}, x_{1}}$ does not coincide with $\sigma$, as the following example shows.

Example. Let $\Sigma$ be a surface embedded in $\mathbb{R}^{3}$ obtained by gluing the spherical cap $x^{2}+y^{2}+z^{2}=r^{2}, z>-\frac{\sqrt{3}}{2} r+\epsilon_{z}$ with a cylinder $x^{2}+y^{2}=r^{2} / 4, z<-\frac{\sqrt{3}}{2} r-\epsilon_{z}$, 
by making a smooth transition in the points with coordinate $z \in\left[-\frac{\sqrt{3}}{2} r-\epsilon_{z},-\frac{\sqrt{3}}{2} r+\right.$ $\epsilon_{z}$ ], for some positive $\epsilon_{z}<\frac{\sqrt{3}}{2} r$. Notice that this transition can be made smooth and depending only on the azimuthal angle $\theta$ in a small interval $\left(\frac{5}{6} \pi-\epsilon_{\theta}, \frac{5}{6} \pi+\epsilon_{\theta}\right), \epsilon_{\theta}<\pi / 6$. Only the details of this surface included in the spherical cap with $\theta \leq \pi / 2+\epsilon$, for some small positive $\epsilon<\pi / 6$, will be relevant.

Let $d l^{2}$ be the induced Riemannian metric on $\Sigma$, and fix $q=(r, 0,0) \in \Sigma$. Consdier the natural product (globally hyperbolic) spacetime $M=\mathbb{R} \times \Sigma, g=d t^{2}-d l^{2}$, with natural projection $\pi: M \rightarrow \Sigma$, and the fixed events $x_{0}=(0, q), x_{1}=(2 \pi r, q)$. The timelike curve $\lambda \mapsto(2 \pi r \lambda, q)$ fix a timelike homotopy class $C_{1}\left(:=C_{x_{0}, x_{1}}^{(1)}\right)$. The connecting lightlike geodesic

$$
\sigma_{0}(\lambda)=\left(2 \pi r \lambda, c_{0}(\lambda)\right), \quad c_{0}(\lambda)=(r \cos 2 \pi \lambda, r \sin 2 \pi \lambda, 0), \quad \lambda \in[0,1],
$$

lies in the boundary $\dot{C}_{1}$, In fact, $\sigma_{0}$ can be reached by approximating the part $c_{0}$ with a constant-speed parametrization $c_{\alpha}$ of $\Sigma \cap \Pi_{\alpha}$, where $\Pi_{\alpha} \subset \mathbb{R}^{3}$ is the plane through $q$, orthogonal to the plane $y=0$, which makes an oriented positive angle $\alpha<\pi / 2$ with the plane $z=0\left(c_{\alpha}\right.$ is contained in the region $z>0$ except in the tangent point $\left.q\right)$. However, by letting $\alpha<0$ we can find a second timelike homotopy class $C_{2}$ such that $\sigma_{0} \in \dot{C}_{2}$; of course, $C_{1}$ and $C_{2}$ are contained in the same causal homotopy class $\mathcal{C}$. Notice that $c_{0}$ passes through the antipodal point $-q=(-r, 0,0)$, which is also a conjugate point of $q$; thus, $\sigma_{0}$ also contains a conjugate point.

Fix $q / m>0$ (resp. $q / m<0$ ), and let $F=\mathcal{B} \pi^{*} \Omega=d \omega$ be on $M$, where $\Omega$ is the volume 2-form of $\Sigma$ (with the orientation induced by the outer normal in the spherical cap), and where $\mathcal{B}: \Sigma \rightarrow \mathbb{R}$ is a non-negative (resp. non-positive) function, with $\mathcal{B} \equiv B>0$ (resp. $<0$ ) constant for $\theta \leq \pi / 2$, acid monotonically decreasing (resp. increasing) to 0 for $\theta \in(\pi / 2, \pi / 2+\epsilon]$. The charged-particle action $I_{x_{0}, x_{1}}$ is given by two contributions. The electromagnetic term reads

$$
\frac{q}{m} \int_{\sigma} \omega=\frac{q}{m} \int_{R} \mathcal{B} \Omega
$$

where, without loss of generality, $\sigma(\lambda)=(2 \pi r \lambda, c(\lambda))$ and $\partial R=c$. For a given length $L \leq 2 \pi r$ of $c$ this integral is maximized in $C_{1}$ by the circle $c_{\alpha}$ with length $L$, namely $c^{L}$. Indeed, the maximizer must be a circle in order to maximize the area, and it is tangent to $c_{0}$ since, otherwise, its enclosed surface $R$ would include regions where $\mathcal{B}<B$ (resp. $\mathcal{B}>B$ ). Thus

$$
\left|\frac{q}{m} \int_{\sigma} \omega\right| \leq \frac{q}{m} B A\left[c^{L}\right],
$$

where $A\left[c^{L}\right]$ is the area contained in $c^{L}$. And the equality holds iff $c=c^{L}$ (up to a reparametrization with the same winding number).

The contribution of the length of $\sigma$ in $I_{x_{0}, x_{1}}$ is:

$$
\int_{\sigma} \mathrm{d} s=\int_{0}^{2 \pi r} \sqrt{1-\left(\frac{\mathrm{d} l}{\mathrm{~d} t}\right)^{2}} \mathrm{~d} t \leq 2 \pi r \sqrt{1-\left(\frac{l[c]}{2 \pi r}\right)^{2}},
$$

where $l[c]$ is the length of $c=\pi \circ \sigma$, and the equality holds when the speed of $c$ is constant. We have then

$$
I_{x_{0}, x_{1}}[\sigma] \leq 2 \pi r \sqrt{1-\left(\frac{l[c]}{2 \pi r}\right)^{2}}+\frac{q}{m} B A\left[c^{l[c]}\right],
$$


where the equality holds iff $\pi \circ \sigma=c^{l[c]}$. But in terms of the angle $0 \leq \alpha \leq \pi / 2$ with $c_{\alpha}=c^{l}$, we have $l=2 \pi r \cos \alpha$ and $A\left[c^{l}\right]=2 \pi r^{2}(1-\sin \alpha)$. Hence if $\frac{q}{m} \overline{B r}>1$,

$$
I_{x_{0}, x_{1}}[\sigma] \leq 2 \pi r^{2} \frac{q}{m} B+2 \pi r\left(1-\frac{q}{m} B r\right) \sin \alpha \leq 2 \pi r^{2} \frac{q}{m} B=I_{x_{0}, x_{1}}\left[\sigma_{0}\right],
$$

and the equality holds iff $\alpha=0$ and the projection of $\sigma$ is $c_{0}\left(=c^{2 \pi r}\right)$, i.e. iff $\sigma=\sigma_{0}$.

Nevertheless, even though $\sigma_{0}$ maximizes in $\bar{C}_{1}$, it does not maximize in $\bar{C}_{2}$ (nor in the causal homotopy class $\mathcal{C}$ ), in agreement with our results. 